\title{
Adenoviral MUC1 Vaccine ETBX-061
}

National Cancer Institute

\section{Source}

National Cancer Institute. Adenoviral MUC1 Vaccine ETBX-061. NCI Thesaurus. Code C143035.

A therapeutic cancer vaccine composed of a replication-defective, serotype 5 adenovirus (Ad5) with the viral genes early 1 (E1), early 2 b (E2b), and early 3 (E3) deleted, and the human glycoprotein mucin 1 (MUC1) encoded, with potential immunostimulating and antineoplastic activities. Upon subcutaneous administration, the adenoviral MUC1 vaccine ETBX-061 expresses the MUC1 protein. The expressed MUC1 may induce a cytotoxic Tlymphocyte (CTL)-mediated immune response against tumor cells expressing MUC1, thereby resulting in both immune-mediated inhibition of tumor cell proliferation and tumor cell death. Deletion of the E1, E2b and E3 genes from Ad5 prevents antiadenovirus immune responses. MUC1, a tumor-associated antigen (TAA) and type I transmembrane protein, is overexpressed in a variety of tumor types. It plays an important role in cancer progression and metastasis. 